\title{
Unification of frequentist inference and machine learning for pterygomaxillary morphometrics
}

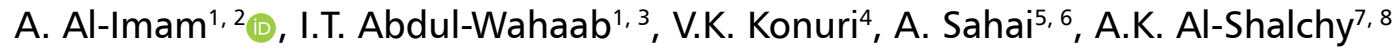 \\ ${ }^{1}$ Department of Anatomy and Cellular Biology, College of Medicine, University of Baghdad, Iraq \\ ${ }^{2}$ Queen Mary University of London, the United Kingdom \\ ${ }^{3}$ Department of Radiology, College of Medicine, University of Baghdad, Iraq \\ ${ }^{4}$ Department of Anatomy, All India Institute of Medical Sciences, Raipur, Chhattisgarh, India \\ ${ }^{5}$ Dayalbagh Educational Institution, Deemed University, Dayalbagh, Agra, India \\ 'International Federation of Associations of Anatomists, Seattle, United States of America \\ ${ }^{7}$ Neurosurgical Unit, Department of Surgery, College of Medicine, University of Baghdad, Iraq \\ ${ }^{8}$ The Royal College of Surgeons, United Kingdom
}

[Received: 17 September 2020; Accepted: 24 November 2020; Early publication date: 30 December 2020]

Background: The base of the skull, particularly the pterygomaxillary region, has a sophisticated topography, the morphometry of which interests pathologists, maxillofacial and plastic surgeons. The aim of the study was to conduct pterygomaxillary morphometrics and test relevant hypotheses on sexual and laterality-based dimorphism, and causality relationships.

Materials and methods: We handled 60 dry skulls of adult Asian males (36.7\%) and females (63.3\%). We calculated the prime distance $D$ [prime] for the imaginary line from the maxillary tuberosity to the midpoint of the pterygoid process between the upper and the lower part of the pterygomaxillary fissure, as well as the parasagittal $D$ [ $x-y$ inclin.] and coronal inclination of $D$ [ $x-z$ inclin.] of the same line. We also took other morphometrics concerning the reference point, the maxillary tuberosity. Results: Significant sexual as well as laterality-based dimorphism and bivariate correlations existed. The univariate models could not detect any significant effect of the predictors. On the contrary, summative multivariate tests in congruence with neural networks, detected a significant effect of laterality on D [ $x$-y inclin.] $(p$-value $=0.066$, partial eta squared $=0.030)$, and the interaction of laterality and sex on $D$ [ $x$ - $z$ inclin.] ( $p$-value $=0.050$, partial eta squared $=0.034$ ). $K$-means clustering generated three clusters highlighting the significant classifier effect of $D$ [prime] and its three-dimensional inclination.

Conclusions: Although the predictors in our analytics had weak-to-moderate effect size underlining the existence of unknown explanatory factors, it provided novel results on the spatial inclination of the pterygoid process, and reconciled machine learning with non-Bayesian models, the application of which belongs to the realm of oral-maxillofacial surgery. (Folia Morphol 2021; 80, 3: 625-641)

Key words: artificial intelligence, cerebral dominance, laterality, masticatory apparatus, osteology, pterygoid process, pterygopalatine fossa, stomatognathic system 


\section{INTRODUCTION}

The pterygomaxillary region and the maxillary alveolus are unique from the anatomical and clinical standpoint and can be affected by several pathologies $[40,46]$. Besides, these regions have peculiar biomechanical properties, blood supply, as well as lymphatic drainage [36]. They neighbour and communicate with some critical areas, including middle cranial fossa, the pterygopalatine fossa, the adjacent part of the base of the skull, the carotid sheath, the nasopharynx and the "danger triangle" of the face [25]. Following a posterior maxillary molar tooth extraction, the management routinely involves dental prostheses and implants. The implant site may require bone grafting techniques that involve the maxillary tuberosity and the pterygoid process of the sphenoid bone, to ensure an implant that closely resembles the normal anatomy and attains biomechanical stability [2]. The clinical applications for studies on morphometry and pathologies affecting the pterygomaxillary junction are valuable for traumatology physicians, oral and maxillofacial surgeons, plastic surgeons and dentists $[2,11]$.

The configuration of the skull is mainly determined by the development of the brain and the masticatory apparatus and the relationships between the brain capsule and the latter [26]. In mammals, the cranial cavity grows with the enlargement of the brain and approaches the nasal cavity [14]. In humans, the nasal cavity altogether with the facial part of the skull, moves under the braincase, not only because of the evolutionary expansion of cerebral hemispheres but also due to the reduction of the masticatory apparatus [27]. These features distinguish the human skull from the skulls of the lowest mammals as well as anthropoid apes [13]. In animals, the sphenoid bone forms as the result of the fusion of several bones that coexist independently [34]. It, therefore, develops as a mixed bone from several paired and unpaired foci of ossification merging at the time of birth into three parts, which, in turn, fuse to form a single bone by the end of the first year of neonatal life [30].

The primary objective of the current study is to measure several morphometric parameters for the pterygomaxillary region, considering various anatomical landmarks. Morphometry will principally include measuring the distance from the maxillary tuberosity to the midpoint of the pterygoid process located between the upper and the lower part of the pterygomaxillary fissure. We shall also estimate the posterior-superior-medial three-dimensional (3D) inclination, i.e., the parasagittal ( $x-y$ plane) and the coronal ( $x-z$ plane) inclination, of the line connecting both points. Morphometry will also include other covariables, including the distance from the maxillary tuberosity to the pterygoid hamulus, the greater palatine foramen, the centre of the cruciform suture, foramen ovale, and foramen spinosum. We shall implement non-Bayesian statistical inference and machine learning models to test three hypotheses. The first two will examine the potential sexual dimorphism and laterality-based differences in connection with our morphometry. The third hypothesis will attempt to explore patterns of causations and the interaction effect among different explanatory factors with a particular interest in explaining the variables affecting the $3 \mathrm{D}$ inclination of the pterygoid process relative to the maxillary tuberosity. We will also discuss the relevant evolutionary lines of the skull, clinical applications in oral and maxillofacial surgery, as well as reporting anecdotal pathologies of interest. Following a pragmatic review of the literature, we confirm that our study is first of its kind regarding its objective and methodology.

We opine that the present study is novel for five reasons. Firstly, our study is the only one in the literature to address the spatial inclination of the pterygoid process in relation to the maxillary tuberosity within the Asian population. Secondly, we are approaching our research question using an unprecedented methodology, in anatomical sciences, by implementing and contrasting classical non-Bayesian statistics versus machine learning techniques. Thirdly, we implemented several models of regression analytics, multivariate tests, and neural networks to attempt finding the model that best describe and explain the pterygomaxillary morphometrics of interest. Fourthly, we argue that this robust approach in analysing data is externally valid and replicable by other researchers, thereby, serving as a foundation of future research within the discipline of anatomical and morphological sciences. Finally, we are analysing our research question and study objectives not only from an anatomical sciences perspective but also from the viewpoint of evolutionary biology and comparative anatomy, which renders our research genuinely interdisciplinary, combining aspects of human anatomy, vertebrates' evolution, data science, and artificial intelligence. 


\section{MATERIALS AND METHODS}

Ethics and code of conduct, the osteology sample, morphometry and tools

The study was conducted following the standard protocol of Ethics and Scientific Committee of the College of Medicine (study protocol 155-19 on 29 December 2019), the declaration of Helsinki by World Medical Association, the European Union (EU) protocol on protection of animals used for scientific purposed (EU Directive 210/63/EU), and the ethical principles of Framingham consensus of 1997.

We used a convenience sampling method and calculated the optimal sample size using IBM SPSS version 24 . Following the exclusion of defective specimens, we collected 60 normal dry skulls $(n=60)$ from the Department of Anatomy. These skulls belonged to adults, males $(n 1=22,36.7 \%)$ and females ( $\mathrm{n} 2=38,63.3 \%$ ) (male-to-female ratio $1: 1.73$ ), of the Asian ethnicity who had no evident pathologies and had an intact base of the skull. Adulthood was determined by the overall ossification and the status of sutures [49]. We also determined the gender (sex) for each skull using a combination of established criteria from the existing literature on the Asian population, by relying on specific anthropometrics, including the mastoid process measurement [32], features of the temporal bone [29], other craniometric traits and parameters of exocranial surfaces [37, 42], the size and shape of the foramen magnum [48], and the external morphology of the frontal bone and frontal sinuses $[20$, $24,32,37,42,48]$. We did our measurements after fixing each skull on a rigid manoeuvrable platform, and fine adjusting the 3D axes (x-y-z) to a standard anatomical position in which the Frankfort horizontal plane is parallel to the ground.

We used Neiko 01407A electronic Vernier calliper, which is accurate in measuring the nearest percentile of a millimetre. We measured two-dimensional (2D) morphometric parameters at the base of the skull, including the prime measurement of the distance from the maxillary tuberosity to the midpoint of the pterygoid process located between the upper and the lower part of the pterygomaxillary fissure (D [prime]). We did not choose any other landmark on the pterygoid process to measure the 3D inclination due to the high variation in the shape as well as the spatial orientation of the sphenoid bone as a whole, including the medial and lateral pterygoid plates, pterygoid hamulus, and processus tubarius. We also recorded the distance from the maxillary tuberosity to the pterygoid hamulus ( $D$ [hamulus]), the greater palatine foramen ( $D$ [greater palatine foramen]), the centre of the cruciform suture ( $D$ [cruciform suture]), foramen ovale ( $D$ [ovale]), and foramen spinosum (D [spinosum]). We used Neoteck aluminium digital angle finder protractor and a digital protractor application (Toolbox pro v5.4.0), which is accurate to the nearest percentile of a degree. In relation to the vertical line, we measured the parasagittal $(x-y)$ and coronal ( $x-z)$ inclination of $D$ [prime] imaginary line. To measure these angles, we approached the skull from the lateral aspect and towards the midsagittal plane of the skull, and took measurements, in millimetres for distances and degrees for inclinations, for each side of the skull, right and left (DR and DL). To avoid measurement errors and biases, we measured the morphometric parameters for each skull twice, and we recorded the average of these for subsequent data analysis.

\section{Data collection and statistical packages, the Bradford Hill criteria, and our hypotheses}

We tabulated raw data using Microsoft Excel 2016 with the integrated Analysis ToolPak, an Excel addin. We imported the data from Excel using the Statistical Package for the Social Sciences (IBM SPSS version 24) to conduct descriptive and inferential statistics as well as to deploy machine learning to our data array. We are using an alpha $(\alpha)$ value of 0.05 as the threshold for significant inferential statistics, and a beta $(\beta)$ value of 0.20 as the cut-off margin for statistical power.

In 1965, English statistician Austin Bradford Hill proposed criteria to provide evidence for understanding the causality between a predictor and an outcome [45]. Hill mandates the analysis of the strength of association and the effect size, the replicability of the results, the specificity of association, the temporality of causation, the gradient effect relationship, plausibility, coherence and consistency, experimental validation, and analogy [45]. Some authors also add reversibility as an additional element to the criteria $[21,45]$. In correspondence with Bradford Hill criteria of causation, we shall test three hypotheses, some of which stem from the general notion of physics and astrophysics that $3 D$ traits represent a derived feature from 2D traits in space [28]. Hence, the 3D topography of the skull, for instance, the inclination of $\mathrm{D}$ [prime], are secondary to the basic 2D morphometric parameters that we measured. 
For instance, the spatial inclination of $D$ [prime] represents a manifestation of $D$ [prime] itself. On the other hand, D [prime] is also affected by the inclination. Therefore, a bidirectional interaction exists between these two parameters. Our first and second hypotheses will test the effect of laterality and sex, i.e., right versus left and males versus females, respectively, on all the parameters that we measured. In the third hypothesis, we shall test $D$ [prime] and its 3D inclination, D [ $x-y$ inclin.] and D [x-z inclin.], as outcome variables while considering sex and laterality as predictors, and controlling for other morphometric parameters as covariates using three models of univariate and multivariate analysis of variance. Using a form of artificial general intelligence, we shall deploy collateral artificial neural networks (supervised machine learning), in addition to clustering analysis (unsupervised machine learning) [4, 35, 39].

\section{Validity and the level-of-evidence}

We evaluated the level-of-evidence according to the Oxford Centre for Evidence-based Medicine (OCEBM) [24]. According to the OCEBM, our study represents an amalgam of an observational cross-sectional data analytic of the osteology sample and an internet snapshot for the systematic review of databases of literature [24, 38].

\section{RESULTS}

\section{Descriptive statistics}

The total sample size was $60(n=60)$ distributed into males and females ( $\mathrm{n} 1=22$ [36.7\%], $\mathrm{n} 2=38$ $[63.3 \%]$, male-to-female ratio $=1.73$ ). The mean, the standard error, skewness, and kurtossis for $D$ [prime] was $10.76,0.24,0.330$, and $0.395, \mathrm{D}$ [x-y inclin.] was $13.23,0.47,0.120$, and $0.058, D$ [ $x-z$ inclin.] was $10.70,0.43,0.552$, and -0.025$), D$ [hamulus] was $12.32,0.17,0.133$, and -0.245 , D [greater palatine foramen] was $13.59,0.19,-0.266$, and -0.243 , D [cruciform suture] was $29.44,0.23,0.074$, and -0.530 , D [ovale] was $33.89,0.30,-0.674$, and 0.618 , and $D$ [spinosum] was $34.88,0.37,-0.117$, and -0.024 . We also explored descriptive statistics while stratifiing the sample by sex and laterality (Table 1 ).

\section{Hypothesis 1: Sexual dimorphism}

Firstly, when comparing in between males and females, there were no statistically significant differences for all the variables except for $D$ [greater palatine foramen] and in favour of the male group
( $\mathrm{t}=2.689, \mathrm{df}=118, \mathrm{p}$-value $=0.008$, mean difference $=1.04,95 \%$ confidence interval $[\mathrm{Cl}]=0.28-1.81$, Cohen's $d=0.52)$. Two variables, $D[x-z$ inclin.] and $D$ [ovale], did not meet the assumptions for running an independent t-test, and therefore, we ran a Mann-Whitney $U$ test. Nevertheless, there was no statistically significant difference between the males and females concerning these two non-normally distributed variables.

Secondly, we stratified the sample based on laterality while comparing males versus females, and we retrieved supplementary results. For the right side of the skull, there was no statistically significant difference between males and females except for $D$ [greater palatine foramen] and in favour of males ( $\mathrm{t}=2.20, \mathrm{df}=52.53, \mathrm{p}$-value $=0.032$, mean difference $=1.29,95 \% \mathrm{Cl}=0.12-2.45$, Cohen's $d=0.61)$. For the left side of the skull, there was only a statistically significant difference for $D$ [spinosum] and in favour of males as well ( $t=2.69$, $\mathrm{df}=58, \mathrm{p}$-value $=0.009$, mean difference $=2.60,95 \%$ $\mathrm{Cl}=0.67-4.53$, Cohen's $\mathrm{d}=0.73$ ). We infer that the skulls of males and females are sexually dimorphic for a few morphometric parameters, including the distance between the maxillary tuberosity and the greater palatine foramen for both sides of the skull, and the distance from the maxillary tuberosity to foramen spinosum for the left side of the skull only. All sexually dimorphic parameters had a medium effect size and in favour of males. Accordingly, causation may potentially exist between sex and the morphometric parameters of the pterygomaxillary region. Therefore, we are going to incorporate sex as an independent variable, within our three models of univariate and multivariate tests as well as neural networks, for testing the third hypothesis.

\section{Hypothesis 2: Laterality-based dimorphism}

Firstly, we compared the right versus the left side of the skull and found no statistically significant difference for all the variables with an exception for $D$ [x-y inclin.] in favour of the left side of the skull ( $\mathrm{t}=-2.54$, df $=118$, $\mathrm{p}$-value $=0.012$, mean difference $=-2.35,95 \% \mathrm{Cl}=-4.19$ to -0.52 , Cohen's $d=0.46$ ), and $D$ [hamulus] in favour of the right side of the skull ( $t=3.060$, $d f=118, p$-value $=0.003$, mean difference $=1.02,95 \% \mathrm{Cl}=0.33-1.67$, Cohen's $d=0.56)$. Besides, the variable $D[x-z$ inclin.] and $D$ [ovale] did not satisfy the prerequisites of an inde- 
Table 1. Descriptive statistics: Stratification by sex and laterality

\begin{tabular}{|c|c|c|c|c|c|c|c|}
\hline \multirow[t]{2}{*}{ Sex } & \multirow[t]{2}{*}{ Laterality } & \multicolumn{2}{|c|}{ Mean } & \multicolumn{2}{|c|}{ Skewness } & \multicolumn{2}{|c|}{ Kurtosis } \\
\hline & & Statistic & $\begin{array}{c}\text { Standard } \\
\text { error }\end{array}$ & Statistic & $\begin{array}{c}\text { Standard } \\
\text { error }\end{array}$ & Statistic & $\begin{array}{c}\text { Standard } \\
\text { error }\end{array}$ \\
\hline \multicolumn{8}{|c|}{ Female } \\
\hline \multicolumn{8}{|l|}{ Left } \\
\hline & D [prime] & 11.1642 & 0.49963 & 0.487 & 0.383 & 0.502 & 0.750 \\
\hline & $\mathrm{D}[\mathrm{x}-\mathrm{y}$ inclination $]$ & 14.4336 & 0.86614 & -0.367 & 0.383 & 0.800 & 0.750 \\
\hline & $\mathrm{D}[\mathrm{x}-\mathrm{z}$ inclination $]$ & 11.7788 & 0.75495 & 0.639 & 0.383 & -0.337 & 0.750 \\
\hline & $\mathrm{D}$ [hamulus] & 11.8062 & 0.25373 & -0.097 & 0.383 & -0.941 & 0.750 \\
\hline & $\mathrm{D}$ [greater palatine foramen] & 13.3338 & 0.32180 & -0.362 & 0.383 & 0.478 & 0.750 \\
\hline & $\mathrm{D}$ [cruciform suture] & 29.0013 & 0.39891 & 0.201 & 0.383 & 0.610 & 0.750 \\
\hline & $\mathrm{D}$ [ovale] & 33.4967 & 0.68618 & -0.502 & 0.383 & -0.123 & 0.750 \\
\hline & $\mathrm{D}$ [spinosum] & 34.6055 & 0.60021 & -0.089 & 0.383 & -0.628 & 0.750 \\
\hline \multicolumn{8}{|c|}{ Right } \\
\hline & D [prime] & 10.1075 & 0.36145 & 0.180 & 0.383 & -1.032 & 0.750 \\
\hline & $\mathrm{D}[\mathrm{x}-\mathrm{y}$ inclination $]$ & 11.6114 & 0.72981 & 0.214 & 0.383 & 0.322 & 0.750 \\
\hline & $\mathrm{D}[\mathrm{x}-\mathrm{z}$ inclination $]$ & 9.4439 & 0.71725 & 0.969 & 0.383 & 0.290 & 0.750 \\
\hline & $\mathrm{D}$ [hamulus] & 12.8698 & 0.35227 & 0.113 & 0.383 & -0.385 & 0.750 \\
\hline & $\mathrm{D}$ [greater palatine foramen] & 13.0736 & 0.38045 & 0.082 & 0.383 & -0.637 & 0.750 \\
\hline & $\mathrm{D}$ [cruciform suture] & 29.6582 & 0.42875 & -0.017 & 0.383 & -0.966 & 0.750 \\
\hline & $\mathrm{D}$ [ovale] & 34.5466 & 0.34226 & -0.104 & 0.383 & -0.970 & 0.750 \\
\hline & $\mathrm{D}$ [spinosum] & 34.0616 & 0.71976 & -0.029 & 0.383 & 0.222 & 0.750 \\
\hline \multicolumn{8}{|c|}{ Male } \\
\hline \multicolumn{8}{|l|}{ Left } \\
\hline & D [prime] & 11.0308 & 0.43402 & -0.334 & 0.491 & -0.978 & 0.953 \\
\hline & $\mathrm{D}[\mathrm{x}-\mathrm{y}$ inclination $]$ & 14.3481 & 1.28436 & 0.408 & 0.491 & -0.815 & 0.953 \\
\hline & $\mathrm{D}$ [x-z inclination $]$ & 10.1321 & 0.85152 & -0.315 & 0.491 & 0.649 & 0.953 \\
\hline & $\mathrm{D}$ [hamulus] & 11.8183 & 0.34684 & 0.361 & 0.491 & -1.227 & 0.953 \\
\hline & $\mathrm{D}$ [greater palatine foramen] & 14.1356 & 0.39723 & -0.637 & 0.491 & -0.467 & 0.953 \\
\hline & $\mathrm{D}$ [cruciform suture] & 29.7637 & 0.56540 & -0.211 & 0.491 & -0.413 & 0.953 \\
\hline & $\mathrm{D}$ [ovale] & 32.9981 & 0.76586 & -0.031 & 0.491 & -0.973 & 0.953 \\
\hline & $\mathrm{D}$ [spinosum] & 37.2012 & 0.72911 & 0.161 & 0.491 & 0.595 & 0.953 \\
\hline \multicolumn{8}{|c|}{ Right } \\
\hline & D [prime] & 10.9345 & 0.63520 & -0.038 & 0.491 & 0.340 & 0.953 \\
\hline & $\mathrm{D}[\mathrm{x}-\mathrm{y}$ inclination $]$ & 12.8071 & 0.98168 & -0.329 & 0.491 & 0.521 & 0.953 \\
\hline & $\mathrm{D}$ [x-z ïnclination $]$ & 11.5779 & 1.21917 & 0.276 & 0.491 & -0.140 & 0.953 \\
\hline & $\mathrm{D}$ [hamulus] & 12.7487 & 0.38554 & -0.968 & 0.491 & 1.292 & 0.953 \\
\hline & $\mathrm{D}$ [greater palatine foramen] & 14.3595 & 0.39371 & -0.163 & 0.491 & 0.893 & 0.953 \\
\hline & $\mathrm{D}$ [cruciform suture] & 29.4954 & 0.54688 & 0.270 & 0.491 & -0.776 & 0.953 \\
\hline & $\mathrm{D}$ [ovale] & 34.3200 & 0.49321 & -1.129 & 0.491 & 2.521 & 0.953 \\
\hline & $\mathrm{D}$ [spinosum] & 34.4622 & 0.88644 & -0.071 & 0.491 & -0.012 & 0.953 \\
\hline
\end{tabular}

pendent t-test. Hence, we conducted an additional Mann-Whitney $U$ test. Nevertheless, there was no statistically significant difference between the two groups.
Secondly, we made a stratification of the sample based on sex while contrasting the differences of the right versus the left side of the skull, and we acquired auxiliary results. For males, laterality had 
only a statistically significant differential effect on $D$ [spinosum] and in favour of the left side of the skull $(\mathrm{t}=-2.386, \mathrm{df}=42$, $\mathrm{p}$-value $=0.022$, mean difference $=-2.74,95 \% \mathrm{Cl}=-5.06$ to -0.42 , Cohen's $d=0.72$ ). For females, laterality had a significant differential effect on three variables only, including $D$ [ $x-y$ inclin.] in favour of the left side of the skull $(\mathrm{t}=-2.492, \mathrm{df}=74, \mathrm{p}$-value $=0.015$, mean difference $=-2.82,95 \% \mathrm{Cl}=-5.08$ to -0.57 , Cohen's $d=0.57$ ), $D$ [x-z inclin.] in favour of the left side of the skull as well $(\mathrm{t}=-2.24$, $\mathrm{df}=74$, $\mathrm{p}$-value $=0.028$, mean difference $=-2.34,95 \% \mathrm{Cl}=-4.41$ to -0.26 , Cohen's $d=0.51$ ), and $D$ [hamulus] in favour of the right side of the skull $(t=2.45, d f=74, p$-value $=0.017$, mean difference $=1.06,95 \% \mathrm{Cl}=0.20-1.93$, Cohen's $d=0.56$ ). To summarise, we deduce that there is an evident dimorphism, based on laterality, regarding the parasagittal inclination of $D$ [prime] and for the distance from maxillary tuberosity to the pterygoid hamulus. Further, laterality-based dimorphism exists within males for the distance from maxillary tuberosity to foramen spinosum, and within females for the parasagittal and the coronal inclination of $D$ [prime] as well as for the distance for from the maxillary tuberosity to the pterygoid hamulus. Almost all of these morphometric parameters had a medium effect size.

In contrast to sex-based dimorphism which was fully predominant in males, laterality-based dimorphism was heterogeneous for both sides of the skull, including when stratifying the sample based on sex. Accordingly, causation can exist between laterality and the pterygomaxillary morphometrics. Therefore, we are going to incorporate laterality as another predictor variable within our three models for testing the third hypothesis on causation.

\section{Bivariate correlations and correlation matrices}

Bivariate correlations: The whole sample. We used Pearson correlation for all the morphometric parameters with an exception of the non-normally distributed D [ $x-z$ inclin.] and D [ovale], for which we used nonparametric tests, including Kendall rank correlation and Spearman correlation. However, we interpreted the results in accordance with the Kendall rank correlation due to its robustness with regard to committing a type-1 statistical error. SPSS computed Pearson's $r$ and Kendall's tau-b correlation coefficients, and the statistical significance for each variable, for one side of the skull versus the contralateral side of it, including D [prime] (Pearson's $r=0.220$, p-value
$=0.091$ ), $\mathrm{D}$ [x-y inclin.] (Pearson's $r=-0.064, \mathrm{p}$-value $=0.627$ ), $D$ [ $x-z$ inclin.] (Kendall's tau- $b=0.247$, $p$-value $=0.005$ ), $D$ [hamulus] (Pearson's $r=0.086$, $p$-value $=0.515)$, $D$ [greater palatine foramen] (Pearson's $r=-0.110, p$-value $=0.403$ ), $D$ [cruciform suture] (Pearson's $r=-0.146, p$-value $=0.267$ ), $\mathrm{D}$ [ovale] (Kendall's tau- $\mathrm{b}=0.017$, $\mathrm{p}$-value $=0.848$ ), and $\mathrm{D}$ [spinosum] (Pearson's $r=0.088, \mathrm{p}$-value $=0.504$ ). To summarise, only $D$ [x-z inclin.] had a significant positive correlation but of a weak effect size.

We also made bivariate correlations, as correlation matrices, for each morphometric parameter with the others, i.e., inter-variable correlations. There was only a statistically significant negative correlation between $D$ [prime] and D [hamulus] (Pearson's $r=-0.181$, $\mathrm{p}$-value $=0.048$ ), and a significant positive correlation between $D$ [ovale] and D [greater palatine foramen] (Kendall's tau-b $=0.127$, $p$-value $=0.040$ ), each had a weak effect size.

Bivariate correlations: Stratification by sex. We stratified the sample by sex and calculated the correlation coefficients and the statistical significance for males and females respectively within each group for D [prime] (males: Pearson's $r=-0.050, p$-value $=0.825$; females: Pearson's $r=0.385$, $p$-value $=0.017$ ), $\mathrm{D}$ [ $x-y$ inclin.] (Pearson's $r=0.055, p$-value $=0.807$; Pearson's $r=-0.142$, $p$-value $=0.396)$, $D$ [x-z inclin.] (Kendall's tau-b $=0.229, p$-value $=0.135$; Kendall's tau-b $=0.245$, p-value $=0.031$ ), $\mathrm{D}$ [hamulus] (Pearson's $r=-0.114$, $p$-value $=0.612$; Pearson's $r=0.185$, $p$-value $=0.266)$, $D$ [greater palatine foramen] (Pearson's $r=-0.266$, $p$-value $=0.231$; Pearson's $r=-0.138, p$-value $=0.409), D$ [cruciform suture] (Pearson's $r=-0.233$, $p$-value $=0.297$; Pearson's $r=-0.090, p$-value $=0.592$ ), D [ovale] (Kendall's tau-b $=-0.048, p$-value $=0.756$; Kendall's tau-b $=0.031, p$-value $=0.782$ ), and D [spinosum] (Pearson's $r=0.159$, $p$-value $=0.479$; Pearson's $r=0.037$, $p$-value $=0.825$ ). To summarise, strictly females had a significant positive correlation for two variables only, including $D$ [prime] and D [x-z] inclination, with a weak-to-medium effect size.

Concerning the inter-variable correlations within males, there was only a statistically significant correlation for $D$ [ovale] versus $D$ [greater palatine foramen] of a medium effect size (Kendall's tau-b $=0.338$, $p$-value $=0.001), D$ [spinosum] versus [greater palatine foramen] of a medium effect size of a medium effect size (Pearson's $r=0.471, p$-value $=0.001$ ), $D$ [ $x-y$ inclin.] versus $D$ [greater palatine foramen] of 
a medium effect size (Pearson's $r=0.315, p$-value $=0.037)$, and $D[x-z$ inclin.] versus $D$ [spinosum] of a weak effect size (Kendall's tau-b $=-0.236$, $p$-value $=0.024)$. Concerning the inter-variable correlations within females, there was only a significant negative correlation of a weak effect size for $D$ [prime] versus $D$ [hamulus] (Pearson's $r=-0.228, p$-value $=0.047$ ). To summarise, significant bivariate correlations exist in between few pterygomaxillary morphometric parameters.

\section{Hypothesis 3: Univariate and multivariate statistics}

Model 1A: Univariate analysis of variance without covariates (ANOVA). To test the causality relationship for the third hypothesis via this model, we used a two-factor (two-way) analysis of variance without controlling for any covariates (ANOVA) by feeding the model with $D$ [prime] as the dependent variable, and sex and laterality as the independent variables (model predictors). According to ANOVA corrected model, sex and laterality, as well as the interaction of these two factors, did not have any significant effect on $D$ [prime] $(\mathrm{df}=3, \mathrm{~F}=1.18$, $\mathrm{p}$-value $=0.320$, partial eta squared $=0.030$, adjusted $\left.R^{2}=0.005\right)$. Pair-wise comparison, via Post-hoc testing using Bonferroni correction, also failed to detect a significant difference in connection with $D$ [prime] based on sex (mean difference $=0.35, p$-value $=0.489$ ) and laterality (mean difference $=-0.58$, $p$-value $=0.251$ ). To summarise, model $1 \mathrm{~A}$ predictors did not have any significant effect on $D$ [prime].

Model 1B: Univariate analysis of variance with covariates (ANCOVA). In this model, we used a two-factor analysis of variance with covariates (ANCOVA). Model 1B has the same architecture of model $1 \mathrm{~A}$ except that we added seven covariates, including D [x-y inclin.], D [x-z inclin.], D [hamulus], $\mathrm{D}$ [greater palatine foramen], D [cruciform suture], $D$ [ovale], and D [spinosum]. Albeit controlling for the covariates, the corrected model also failed to detect any significant effect of sex and laterality on $\mathrm{D}$ [prime] ( $\mathrm{df}=10, \mathrm{~F}=1.155$, $\mathrm{p}$-value $=0.329$, partial eta squared $=0.096$, adjusted $R^{2}=0.013$ ). Further, subsequent pairwise comparison, using Bonferroni correction, yielded no significant differences in between males versus females (mean difference $=0.20$, $p$-value $=0.700$ ), and the right side of skull versus the left side of the skull (mean difference $=-0.58$, $p$-value $=0.298$ ). As with model $1 \mathrm{~A}$, the predictors in model $1 \mathrm{~B}$ did not have a significant effect on the outcome, $D$ [prime].
Model 2A: Multivariate analysis of variance without covariates (MANOVA). In model 2A, we will use a three-way multivariate analysis of variance (MANOVA) without covariates. Here, we will study the effect of three independent variables, including sex, laterality, and D [prime] versus the three-dimensional inclination of $D$ [prime] represented by the two dependent variables $D$ [ $x-y$ inclin.] and $D$ [ $x-z$ inclin.]. All the prerequisites to run the multivariate model were satisfied, including Box's test for equivalence of covariance matrices (Box's $M=9.626, F=1.033$, $\mathrm{df1}=9, \mathrm{df2}=65056.903, \mathrm{p}$-value $=0.410)$. Hence, we will interpret the results of the multivariate test using Wilks' lambda. Neither D [prime] nor sex had a significant effect on the outcome. Nonetheless, at an alpha value of 0.10 , laterality assumed a significant effect on the spatial inclination of $D$ [prime] (Wilks' lambda $=0.952, F=2.863$, hypothesis $\mathrm{df}=2$, error $\mathrm{df}=114$, $\mathrm{p}$-value $=0.061$, partial eta squared $=0.048$ ), and the interaction of sex and laterality as well (Wilks' lambda $=0.956, \mathrm{~F}=2.653$, hypothesis $\mathrm{df}=2$, error $\mathrm{df}=114$, $p$-value $=0.075$, partial eta squared $=0.044$ ). Tests of between-subjects effects validated the results of the multivariate tests. Laterality had a significant effect on $D$ [ $x$-y inclin.] $(\mathrm{df}=1, \mathrm{~F}=5.521, \mathrm{p}$-value $=0.020$, partial eta squared $=0.046$ ) but not on $D[x-z$ inclin. $]$. On the contrary, the interaction of sex and laterality had a significant effect on $D$ [ $x-z$ inclin.] ( $d f=1, F=4.945$, p-value $=0.028$, partial eta squared $=0.041$ ) but not on $\mathrm{D}[\mathrm{x}-\mathrm{y}$ inclin.]. To summarise, only two predictors of model 2A had a significant effect on the parasagittal and coronal inclination of $D$ [prime], and each had a weak effect size.

Model 2B: Multivariate analysis of variance with covariates (MANCOVA). In this model, we will run a multivariate analysis of variance while controlling for covariates (MANCOVA) (Box's $\mathrm{M}=9.626$, $\mathrm{F}=1.033$, df1 $=9$, df2 $=65056.903, \mathrm{p}$-value $=0.410$ ). The design is similar to model $2 \mathrm{~A}$ except that we added the rest of the morphometric parameters as covariates. The multivariate model $2 \mathrm{~B}$ failed to infer any significant effect of the predictors in connection with the outcome variables. However, when considering an alpha value of 0.10 for tests of between-subjects effects, there was a significant effect of laterality on $D$ [ $x-y$ inclin.] ( $d f=1, F=3.741$, $p$-value $=0.056$, partial eta squared $=0.033$ ), and a significant effect of the interaction of sex and laterality on $D[x-z$ inclin.] (df $=1, F=4.301$, p-value 
$=0.040$, partial eta squared $=0.038$ ). To summarise, the MANCOVA-based model $2 B$ is harmonious with model 2A. However, model 2B is more accurate in terms of predictive power due to the consideration of covariates in multivariate testing.

Model 3A: Multivariate analysis of variance without covariates (MANOVA). Model 3A will run a two-way multivariate analysis of variance without covariates (MANOVA) (Box's $M=24.177, F=1.274$, $\mathrm{df1}=18, \mathrm{df} 2=28191.950, \mathrm{p}$-value $=0.193)$. We fed the model with laterality and sex as inputs (predictors), and three outcome variables represented by D [prime], D [x-y inclin.], and D [x-z inclin.]. At an alpha value of 0.10 , the multivariate analysis confirmed a significant effect of laterality on $D$ [prime] and its spatial inclination (Wilks' lambda $=0.941$, $\mathrm{F}=2.366$, hypothesis $\mathrm{df}=3$, error $\mathrm{df}=114$, $\mathrm{p}$-value $=0.075$, partial eta squared $=0.059$ ). Tests of between-subjects effects verified the results of the multivariate tests; it is evident that laterality had a significant effect only on $D$ [ $x-y$ inclin.] ( $d f=1$, $F=5.110, p$-value $=0.026$, partial eta squared $=0.042$ ), while the interaction of sex and laterality also had a significant effect but only on $D[x-z$ inclin.] ( $d f=1, F=4.537$, $p$-value $=0.035$, partial eta squared $=0.038$ ). The results of model $3 \mathrm{~A}$ are in line with the previous multivariate models, and each of its predictors had a weak effect size.

Model 3B: Multivariate analysis of variance with covariates (MANCOVA). Model 3B is summative for all the previous three multivariate models. We will use multivariate analysis of variance while controlling for covariates (MANCOVA) (Box's $M=24.177$, $\mathrm{F}=1.274, \mathrm{df1}=18, \mathrm{df2}=28191.950, \mathrm{p}$-value $=0.193$ ). The model has the same layout of the model $3 \mathrm{~A}$, but here, we are also incorporating the rest of the morphometric parameters as covariates. MANCOVA-based multivariate tests of model 3B could not detect any significant effect for the predictors on the outcome variables. However, tests of between-subjects effects at an alpha value of 0.10 , detected a significant effect of laterality on $D$ [ $x-y$ inclin.] ( $d f=1$, $\mathrm{F}=3.443, \mathrm{p}$-value $=0.066$, partial eta squared $=0.030$ ), and another significant effect of the interaction of sex and laterality on $D$ [ $x-z$ inclin.] ( $d f=1$, $\mathrm{F}=3.926$, $\mathrm{p}$-value $=0.050$, partial eta squared $=0.034)$. To recapitulate the multivariate analytics, only laterality, and the interaction of sex and laterality, had a significant effect. The former influenced the parasagittal inclination, while the latter affected the coronal inclination of the imaginary line connecting the maxillary tuberosity to the midpoint of the pterygoid process between the upper and the lower part of the pterygomaxillary fissure. The significant predictor effect persists to be true even when integrating covariates to the multivariate models. However, the effect size remains weak, and none of the predictors had a significant effect on $D$ [prime].

\section{Hypothesis 3: Multiple linear regression}

Model A. In all three regression models, we used dummy coding of sex and laterality to fit the linear nature of the analysis, and each model allowed feeding one dependent variable at a time. Here, we designated $D$ [prime] as the dependent variable while all other morphometric parameters, as well as sex and laterality, represented the independent variables. As per the model A summary, the predictors did not have any significant effect on the outcome $\left(R=0.290, R^{2}=0.084\right.$, adjusted $R^{2}=0.009, R^{2}$ change $=0.084, \mathrm{~F}$ change $=1.121, \mathrm{df} 1=9, \mathrm{df} 2=110$, $\mathrm{p}$-value $=0.354$ )

Model B. In model B, we specified D [ $x-y$ inclin.] as the outcome, while other morphometric parameters and the dummy-coded string variables as the model predictors. Similar to the previous model, model B failed to detect any significant effect of the independent variables in connection with the parasagittal inclination of $D$ [prime] $\left(R=0.290, R^{2}=0.084\right.$, adjusted $R^{2}=0.018, R^{2}$ change $=0.084, F$ change $=1.273$, $\mathrm{df1}=8, \mathrm{df2}=111, \mathrm{p}$-value $=0.265$ ). Nevertheless, "tweaking" the $\alpha$ value for hypothesis testing up to 0.10 , it is apparent that laterality had a significant effect on the parasagittal inclination and in favour of the left side of the skull (standardized beta coeffecient $=0.198, \mathrm{t}=1.968, \mathrm{p}$-value $=0.052$ ).

Model C. The architecture of model $C$ is somewhat similar to the previous model except that we allocated D [ $x-z$ inclin.] as the dependent variable. In correspondence with the model $\mathrm{C}$ summary, multiple linear regression also failed to infer any significant effect of the predictors on the outcome, the coronal inclination of $D$ [prime] $\left(R=0.258, R^{2}=0.067\right.$, adjusted $R^{2}=0.008, R^{2}$ change $=0.067, F$ change $=1.144$, $\mathrm{df1}=7, \mathrm{df2}=112, \mathrm{p}$-value $=0.341)$. Nevertheless, if we "update" the alpha value to $0.10, \mathrm{D}$ [spinosum] will have a significant effect in connection with $\mathrm{D}$ [x-z inclin.] (standardized beta coeffecient $=-0.185$, $\mathrm{t}=-1.912, \mathrm{p}$-value $=0.058)$. We are concluding, based on all three regression models, that only a few 
predictors, including laterality and D [spinosum], had a significant effect on the spatial inclination of $\mathrm{D}$ [prime], and strictly conditioned by manipulating the cut-off margin of the significance level.

\section{Hypothesis 3: Supervised machine learning}

Neural networks: Model 1A. We ran a neural network analysis that is complementary to the earlier univariate analysis of variate without covariates (ANOVA) in connection with model $1 \mathrm{~A}$ for testing the third hypothesis on causality relationship. Here, we used sex and laterality as the input layer (independent variables) and $D$ [prime] as the output layer (dependent variable). Independent variable importance and synaptic weights were superior for laterality (importance $=0.910$, normalized importance $=100 \%$ ) compared to sex (importance $=0.090$, normalized importance $=9.9 \%$ ) in predicting $\mathrm{D}$ [prime].

Neural networks: Model 1B. This model is parallel to the univariate analysis of variance while controlling for covariates (ANCOVA) that we implemented to test the third hypothesis using model 1B. For the input layer, we used sex and laterality as independent variables, while considering $D$ [ $x-y$ inclin.], $D[x-z$ inclin.], D [hamulus], $D$ [greater palatine foramen], D [cruciform suture], D [ovale], and D [spinosum] as covariates. For the output layer, D [prime] represented the dependent variable. As per the independent variable importance analysis, this model allocated the highest values to $D$ [ $x-z$ inclin.] (importance $=0.234$, normalized importance $=100.00 \%), D[x-y$ inclin. $]$ $(0.203,86.70 \%), D$ [hamulus] $(0.193,82.80 \%)$, $\mathrm{D}$ [greater palatine foramen] $(0.132,56.50 \%)$, laterality $(0.064,27.30 \%), D$ [ovale] $(0.06,25.70 \%)$, D [spinosum] $(0.055,23.40 \%), D$ [cruciform suture] $(0.038,16.40 \%)$, and sex $(0.021,9.20 \%)$. Still, it appears that laterality is more important than sex in predicting $\mathrm{D}$ [prime] in aggreement with the nerual network analysis of model 1A. On the other hand, the coronal and the parasagital inclination of $D$ [prime] assumed the highest weights among the covariates.

Neural networks: Model 2A. We implemented neural networks to fulfil the multivariate analysis of variance (MANOVA) for testing the causation in model 2A. We used sex, laterality, and $D$ [prime] as inputs (predictors) versus $D$ [x-y inclin.] and $D$ [ $x-z$ inclin.] as outputs (outcome variables). The model's independent variable importance analysis designated the magnitudes for $D$ [prime] $(0.483,100.00 \%)$, laterality $(0.397,82.20 \%)$, and sex $(0.121,25.00 \%)$. According to this model, the spatial orientation of $D$ [prime] is predicted most accurately by $\mathrm{D}$ [prime] itself, laterality, and sex as well.

Neural networks: Model 2B. This model is in line with the multivariate analysis of variance with covariates (MANCOVA) that we ran to test the causality relationship of model $2 \mathrm{~B}$ of the third hypothesis. The input layer included sex and laterality, as well as six covariates, including D [prime], D [hamulus], D [greater palatine foramen], D [cruciform suture], $D$ [ovale], and $D$ [spinosum] as the explanatory variables. Similar, to the neural network of model 2A, the output layer had $D[x-y$ inclin.] and $D$ [ $x-z$ inclin.] as the response variable. Independent variable importance analysis quantified the highest values for $D$ [prime] $(0.194,100.00 \%), D$ [spinosum] $(0.178,91.50 \%)$, $\mathrm{D}$ [greater palatine foramen] $(0.162,83.10 \%)$, laterality $(0.129,66.30 \%), D$ [ovale] $(0.127,65.10 \%), D$ [hamulus] $(0.120,61.70 \%), D$ [cruciform suture] (0.049, $25.40 \%)$, and sex $(0.041,21.10 \%)$. In harmony, with the neural network of model $2 \mathrm{~A}$, it appears that D [prime], as well as laterality and sex, are important predictors of the parasagittal and coronal inclination of the imaginary line connecting the maxillary tuberosity and midpoint of the pterygoid process located between the upper and the lower part of the pterygomaxillary fissure. However, when controlling for the covariates via this model, it is obvious that the normalized importance of laterality and sex is lower when contrasted with those of model $2 \mathrm{~A}$.

Neural networks: Model 3A. We used a neural network that is supportive of the multivariate statistical analysis (MANOVA) in model $3 \mathrm{~A}$ of the third hypothesis on causation. Here, the inputs had laterality and sex as the only predictors without taking any covariates into account (Fig. 1). The output layer included D [prime], D [ $x-y$ inclin.], and D [x-z inclin.] as the dependent variables. The predictor importance analysis showed different results from the neural networks in the previous four models. Here, the neural network assigned more importance for sex (0.597, $100.0 \%)$ than laterality $(0.403,67.6 \%)$. Nonetheless, this neural network model is still in agreement with our previous results validating that males and females are sexually dimorphic in connection with the morphometry of the pterygomaxillary landmarks.

Neural networks: Model 3B. We are concluding the supervised machine learning analytics with a summative neural network model that is analogous to the multivariate statistical analysis of variance with 

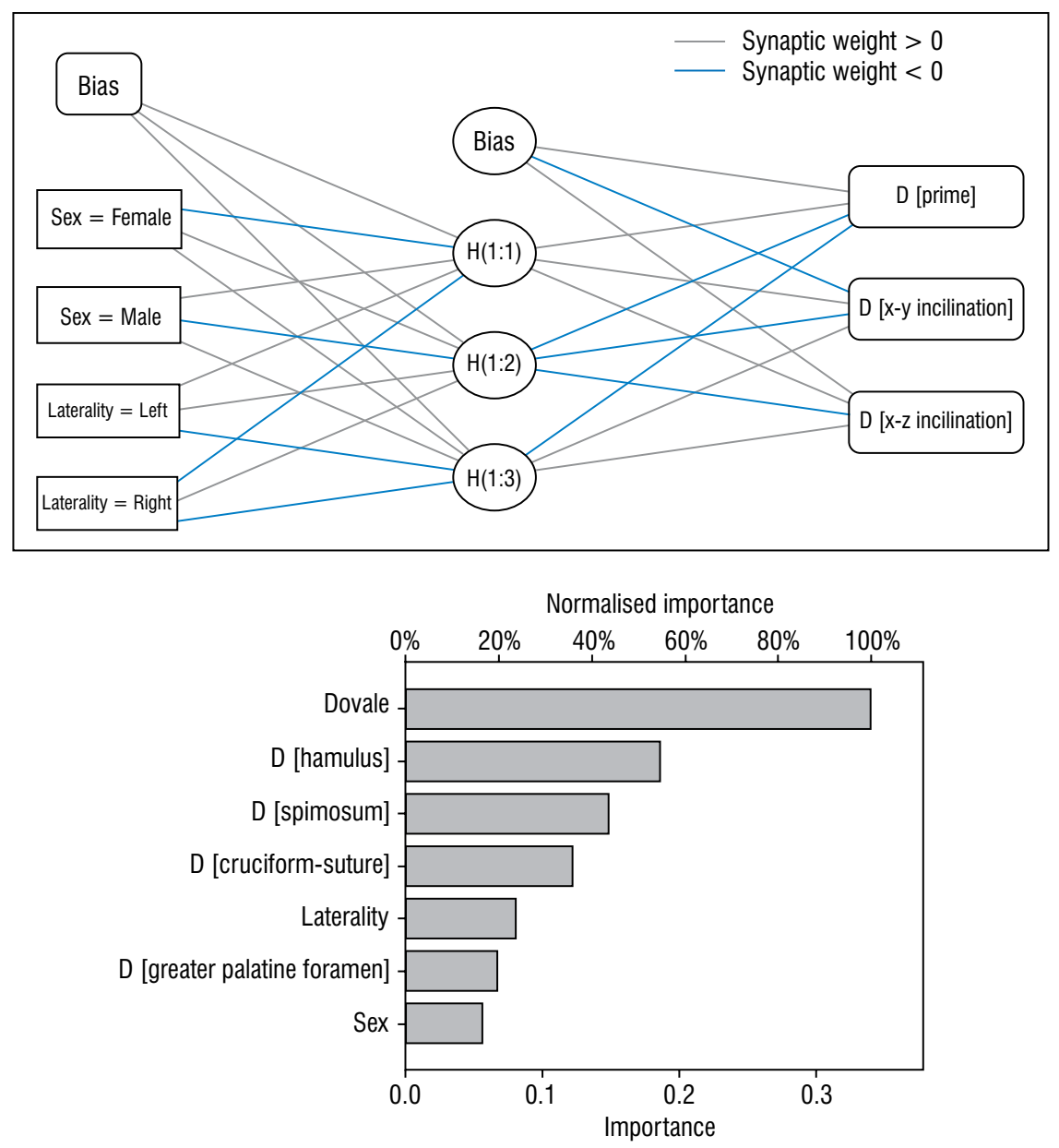

Figure 1. Neural networks with synaptic weights (Model 3a) and independent variable importance analysis (Model 3B).

covariates (MANCOVA) from model 3B of the third hypothesis. Here, the architecture of the network is the same as that of model $3 \mathrm{~A}$ except that we added five covariates to the input layer including $D$ [hamulus], D [greater palatine foramen], D [cruciform suture], D [ovale], and D [spinosum] (Fig. 1). The independent variable importance analysis assigned the highest importance for $D$ [ovale] $(0.34,100.00 \%)$, D [hamulus] $(0.185,54.50 \%), D$ [spinosum] $(0.149$, $43.70 \%)$, D [cruciform suture] $(0.122,35.90 \%)$, laterality $(0.080,23.60 \%), D$ [greater palatine foramen] $(0.067,19.50 \%)$, and sex $(0.056,16.50 \%)$. Here, the predictor importance analysis departs from that of model $3 \mathrm{~A}$ by generating a higher weight for laterality when compared to sex, and in harmony with the rest of the neural networks, which is in agreement that laterality-based dimorphism exists among the morphometric parameters. Among all the models, we opine that model 3B neural network is supreme in predicting $D$ [prime] and its spatial inclination by analyzing the interaction between the several predictors, including covariates, on the outcome variables.

\section{Unsupervised machine learning}

Two-step cluster analysis. We used two-step clustering by feeding the clustering algorithm with sex and laterality as categorical variables, and the morphometric parameters as continuous variables. For the whole sample, the algorithm generated three clusters of decent quality (Silhouette measure of cohesion and separation $=0.3$ out of 1 ). The largest cluster contributed to $36.7 \%$ of the total sample, while each of the other two clusters accounted for $31.7 \%$ (largest cluster to smallest cluster ratio $=1.16$ ). Predictor importance analysis assigned the highest importance for sex (predictor importance $=1$ ), laterality (0.63), $D$ [greater palatine foramen] (0.06), $D$ [hamulus] (0.05), D [x-y inclin.] (0.05), D [x-z inclin.] (0.04), D [spinosum] (0.03), D [prime] (0.03), D [ovale] $(0.02)$, and $D$ [cruciform suture] (0.01). The largest 
cluster included males only (100\%), and each of the other two clusters included females only $(100 \%)$. The predictor importance analysis, cluster comparison, and the cell distribution within each cluster go in line with the earlier results that males and females are sexually dimorphic in addition to the existence of laterality-based dimorphism within skull specimens.

Upon stratifying the sample based on sex, the twosteps cluster analysis generated a total of four clusters, two clusters for each of males and females. For males, the cluster quality was fair (Silhouette measure of cohesion and separation $=0.3$ ), and each cluster had $50 \%$ of the total sample size (largest cluster to smallest cluster ratio $=1$ ). Predictor analysis allocated the importance values for laterality (predictor importance $=1), D$ [spinosum] $(0.16), D$ [hamulus] (0.10), D [ovale] (0.08) D [x-z inclin.] (0.05), D [x-y inclin.] (0.04), $D$ [greater palatine foramen] (0.02), and $D$ [cruciform suture] (0.01). For females, The clustering quality was also similar (average silhouette $=0.3$ ), and each cluster contributed to $50 \%$ of the total sample (cluster ratio $=1$ ). The clustering algorithm designated the highest importance to laterality (predictor importance $=1), D[x-y$ inclin.] (0.10), $D$ [hamulus] (0.10), D [x-z inclin.] (0.09), D [prime] (0.06), D [ovale] (0.04), D [cruciform suture] (0.03), $D$ [spinosum] (0.01), and $D$ [greater palatine foramen] (0.01). The clustering within males and females gave somewhat similar results and corresponded primarily with our earlier results on the existence of a laterality-based dimorphism while assigning relatively higher importance for $D$ [prime] among females.

Stratification of the sample based on laterality also generated four clusters, two for each of the right side of the skull and the left side of the skull, and each cluster was purely made of one of the two sexes, either males or females. For the right side of the skull, the cluster quality was fair (average silhouette $=0.3$ ), and the largest cluster accounted for $63.3 \%$ (cluster size ratio $=1.73$ ). . The algorithm computed the highest importance for sex (predictor importan$\mathrm{ce}=1), \mathrm{D}$ [greater palatine foramen] $(0.11), \mathrm{D}[\mathrm{x}-\mathrm{z}$ inclin.] (0.07), D [prime] (0.05), D [x-y inclin.] (0.03), $\mathrm{D}$ [ovale] (0.01), D [spinosum] (0.01), D [cruciform suture] (0.01), and $D$ [hamulus] (0.01). For the left side of the skull, the clustering parameters were the same as those for the right side. The algorithm calculated the highest importance for sex (predictor importance $=1), D$ [spinosum] $(0.14), D$ [greater palatine foramen] (0.06), D [x-z inclin.] (0.05),

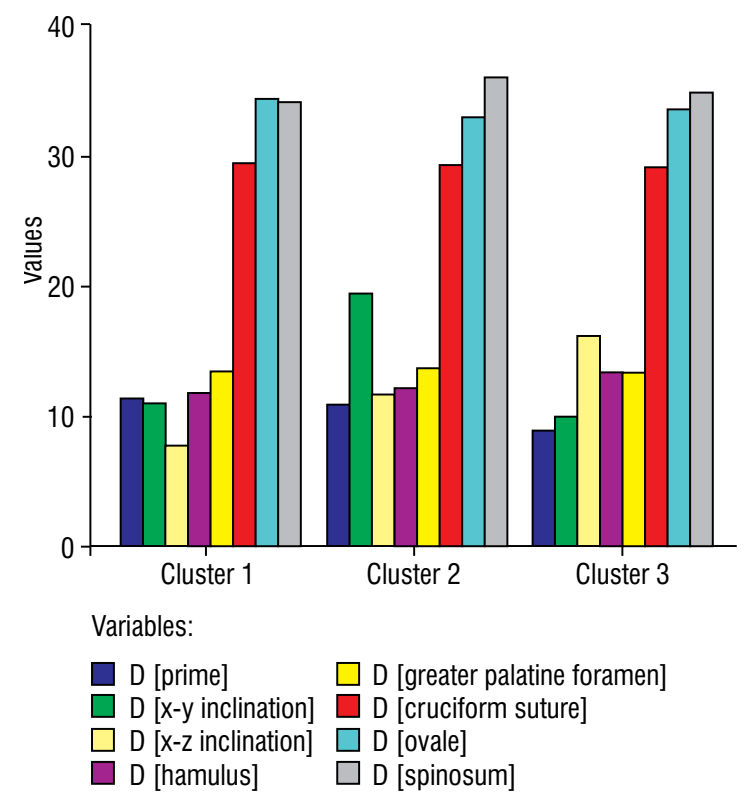

Figure 2. K-means cluster analysis: Final cluster centres.

$\mathrm{D}$ [curicform suture] (0.04), and D [ovale] (0.01). Finally, the stratification of the sample based on both sex and laterality together failed to yield any clusters. We conclude that the two-step cluster analysis validates our preexisting results, from independent t-tests and the multivariate models as well as the neural networks, on sex and laterality-based dimorphism in connection with the pterygomaxillary junction morphometrics.

K-means and hierarchical cluster analysis. In SPSS, k-means clustering did not allow to feed string variables to the model, including sex and laterality, and therefore, the results of this algorithm are unique from those of the two-step cluster analysis, as it relied strictly on feeding the model with continuous variables. The k-means clustering yielded three distinguished clusters (Fig. 2), and calculated the final cluster centres for $D$ [prime] $\left(1^{\text {st }}\right.$ cluster $=11.39$, $2^{\text {nd }}$ cluster $=10.94,3^{\text {rd }}$ cluster $\left.=9.01\right), D[x-y$ inclin. $]$ $(11.05,19.47,10.03), D[x-z$ inclin.] (7.81, 11.82, 16.24), $D$ [hamulus] $(11.90,12.23,13.46), D$ [greater palatine foramen] $(13.57,13.72,13.45), \mathrm{D}$ [cruciform suture] $(29.58,29.38,29.19)$, D [ovale] $(34.48,33.03$, $33.60)$, and $D$ [spinosum] $(34.17,36.11,34.94)$. ANOVA testing confirmed that only four morphometric parameters significantly differed among the three clusters, including $D$ [prime] (cluster $\mathrm{df}=2$, error $\mathrm{df}$ $=117, F=8.135, p$-value $<0.001), D[x-y$ inclin. $]$ (cluster $\mathrm{df}=2$, error $\mathrm{df}=117, \mathrm{~F}=82.390, \mathrm{p}$-value $<0.001$ ), $D$ [x-z inclin.] (cluster $d f=2$, error $d f=117$, 
$\mathrm{F}=56.875, \mathrm{p}$-value $<0.001)$, and $\mathrm{D}$ [hamulus] (cluster $\mathrm{df}=2$, error $\mathrm{df}=117, \mathrm{~F}=6.827, \mathrm{p}$-value $=0.002$ ). Compared with the neural network from model 3B, our k-means clustering algorithm conveyed novel results in connection with the importance of $D$ [prime] and its 3D inclination in classifying our sample regardless of sex and laterality for each specimen. We concluded the clustering analysis by conducting a hierarchial clustering which was also successful in creating a dendrogram of multiple levels.

\section{DISCUSSION}

Concerning our first hypothesis, significant sexual dimorphism existed in favour of males, and as per our pre-study anticipation. However, these were in connection with two morphometric parameters only, including $D$ [greater palatine foramen] and $D$ [spinosum]. For the second hypothesis, significant laterality-based dimorphism was more diversified as it did not favour one side of skull even when stratifying the sample based on sex, and it involved more morphometric parameters including $D[x-y$ inclin.], D [x-z inclin.], D [hamulus], and D [spinosum]. Each of sexual and laterality-based dimorphism had a medium effect size. Further, significant intra-variable and inter-variable correlations existed for the whole sample and within males and females as well. However, contrary to our expectations, these correlations involved few morphometric parameters and had a weak-to-medium effect size. Testing the third hypothesis using the univariate analysis of variance and multiple linear regression failed to detect any significant predictors. In contrary, multivariate statistical models were triumphant in elucidating the significant effect of laterality, and the interaction of sex and laterality on the parasagittal and coronal inclination of D [prime], respectively. However, the weak effect size for those explanatory variables entails the existence of covert predictors, and perhaps an abundance of them interacting to manifest the full variance within the outcome. Supervised and unsupervised machine learning, using neural networks and clustering analysis, reconciled with the non-Bayesian statistical models, especially the multivariate tests, and across all of our three hypotheses. Besides, k-means cluster analysis highlighted the significant classifier effect of $D$ [prime], its three-dimensional inclination, and D [hamulus].

Concerning the evolutionary lines of the skull, the interrelationship between the brain and the masticatory apparatus determined the overall morphology of the skull $[13,27,30]$. At first, the orbit, the nasal, and the oral cavities were located in front of the cranium $[13,26]$. With the enlargement of the brain in mammals, the cranial cavity approached the nasal cavity $[19,34]$. In man, the nasal cavity and the facial skeleton moved under the braincase (cranium), not only because the brain is enlarged but also because the masticatory apparatus is minimised $[14,26,27,30]$. The singularity of the sphenoid bone also emerged because of the fusion of several independent bones that still exist in animals [30]. Therefore, it developed as a mixed bone from several paired and unpaired ossification foci merging at the time of birth into three parts, which, in turn, form a single bone by the end of the first year of life [30]. Throughout evolution, the number of teeth got reduced, and the third molar is still undergoing evolutionary pressure to disappear in humans $[13,14,26,30]$. Further, the bulging of the forehead and the receding of the snout gave rise to the delicate proportions of the human face [13]. These anatomical features distinguished the human skull from those of lower mammals as well as the nearest anthropoid apes [19, 34]. The evolution of the craniofacial complex took place through natural selection, and yet it is constrained by the interplay of the genotype and phenotype [13, 19, 27]. If "modules" are understood as localised areas of genotypic and phenotypic integration, then we need to define the spatio-temporal boundaries of these modules and enumerate their attributes and testable properties [13, 14]. In 2006, Polanski and Franciscus (2006) [41] pointed out that "evolutionary biology has long maintained that morphological systems go from being more integrated to more modularised throughout evolution" [41]. Hence, intricate anatomical structures like the vertebrate skull become even more modularised [13, 19, 26, 27, 30, 34]. Scholars also refer to "heterochrony" which describes the evolution of ontogeny by modifications in the rate and timing of essential constituents of ontogenetic trajectories, such as the onset, the offset, and the rates of growth and development [51, 52]. Thus, causality can be questionable for craniofacial ontogeny as it is a four-dimensional process encompassing a temporal axis (time), and most evolutionary modifications of development may cause some deviations in the course of events along that axis [30,34]. Nevertheless, developmental processes others than heterochrony, namely spatial integration, dissociation, and constraints do happen during evolution $[14,51,52]$. 
The pterygoid plates assume a crucial function from a biomechanical perspective in connection with the stomatognathic system, specifically the masticatory apparatus, palatal muscles, and the pharyngeal constrictors [31]. Several muscles attach to or operate via, the maxillary tuberosity and the pterygoid process of the sphenoid bone, including the buccinator, the pterygoid muscles, levator veli palatini (levator palati), and tensor veli palatine (tensor palati) [31]. Likewise, the pterygoid hamulus mimics Archimedean mechanical systems, having a pulley-like structure for the tensor palati, while the hamulus itself anchors a critical mechanical structure, the pterygomandibular raphe (pterygomandibular ligament) [31, 43]. Besides, several notable neurovascular structures exist in the vicinity of the pterygoid process, primarily the mandibular branch of the trigeminal nerve, the middle meningeal artery, nervus spinosus, the petrosal nerves, as well as the neurovascular elements of the vidian canal [31, 43]. Accordingly, the current study is of prime importance for medicine, surgery, and biomimetics. Implant dentistry specialists also do have interests in the pterygomaxillary junction, which grew exponentially following the introduction of the osseointegration theory by Branemark in the early 1960s [see 1]. Researchers found that rehabilitation of missing teeth in the anterior segment of the maxillary alveolus is far more straightforward than the posterior segment [1]. Reconstruction of the posterior maxilla is a challenge due to several obstacles, some of which relate to the anatomy of the maxillary sinus, and numerous surgical procedures were tried, such as bone augmentation, sinus lift, tilted and short implants, and zygomatic implants [1, 12]. Each of these procedures has restrictions, while the pterygomaxillary region provided an excellent venue for infallible rehabilitation of the posterior maxilla [12]. Balaji et al. [12] described using pterygoid implants for restoring an atrophic posterior maxilla, and they have higher success rates, fewer complications, and better acceptance by patients to manage atrophic posterior maxilla when compared to conventional implants [12]. In 2012, Candel et al. [18] referred to two anatomical sites for retromolar implants, including the pterygoid process and the pterygomaxillary region. According to the systematic review by Bidra and Huynh-Ba (2011) [15], the cumulative survival rate for those implants over ten years, and based on data from one of the studies was $91 \%$.
On the other hand, the maxillary tuberosity is nearby the third molar, and it relates to a critical condition in oral and maxillofacial surgery, alveolar osteitis, which is the most frequent postoperative complication following a tooth extraction, especially for an impacted third molar nearby the maxillary tuberosity $[2,15,17,22]$. Crawford [22] was the first to describe the condition in 1896. In their meta-analytic study, Bienek and Filliben (2016) [16] declared that the use of oral contraceptives significantly increased the risk of developing alveolar osteitis. Taberner-Vallverdú et al. (2016) [47] wrote on the heterogeneity of therapeutic modalities for alveolar osteitis, including curettage and irrigation, antiseptics, low-level laser therapy, zinc oxide eugenol, and platelet-rich plasma. Marcussen et al. (2016) [33] found that a single preoperative oral dose of amoxicillin or penicillin- $V$ substantially reduced the incidence of alveolar osteitis. From 2012 to 2016, chlorhexidine was the centre of several metareviews. In 2012, Yengopal and Mickenautsch [50] inferred that chlorhexidine had no more adverse reactions compared to a placebo. In the same year, Daly et al. [23] validated that rinsing the alveolar sockets with chlorhexidine $(0.12 \%$ and $0.2 \%)$ or using chlorhexidine gel $(0.2 \%)$, prevented alveolar osteitis. Sánchez et al. (2017) [44] concluded that chlorhexidine, in any formulation, was also useful for prophylaxis, although the gel was more efficacious. Zhou et al. (2017) [53] deduced the same on the higher efficacy of chlorhexidine gel.

The current study does have limitations, including the sample size, which is relatively small. Other parameters that are unique for the sample cannot be fully known, for example, the exact age, the sub-ethnicities of individuals to whom the skulls belong. Besides, other demographic variables are obscure, including the patterns of cerebral dominance for each individual, the existence of underlying pathologies affecting the skeletal system or other corporeal systems in the premortem. Finally, there are some limitations of statistical analyses. For instance, the immoderate type- 1 statistical error that may manifest as a consequence of carrying out multiple data analytics. Some tests, including correlation analytics, were more conservative than others as in the case of Kendall rank correlation versus Spearman correlation, and the same applies to parametric tests when compared to nonparametric tests. Additionally, the interpretation of causality in our hypotheses and different models that we implemented may accept 


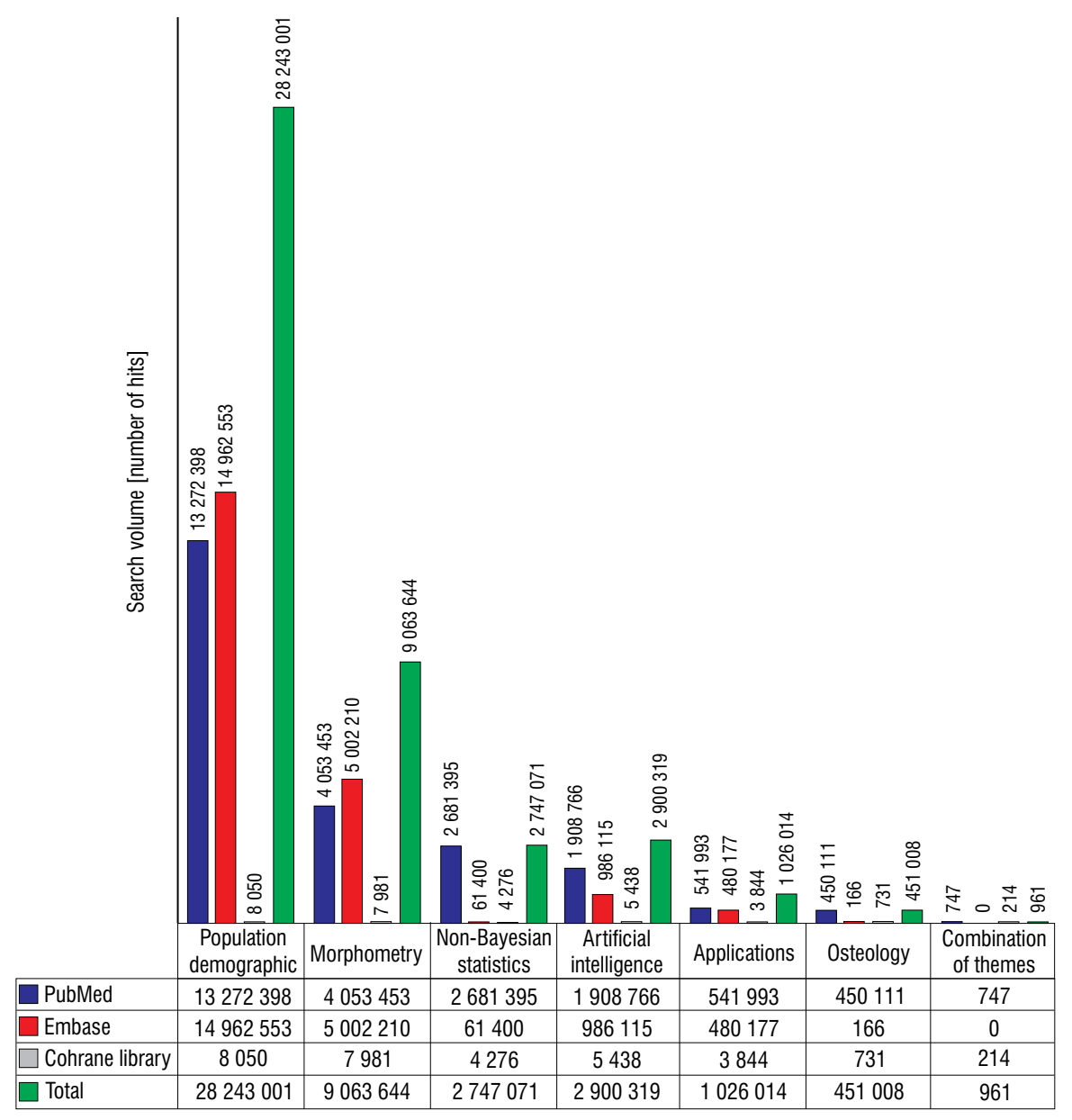

Figure 3. Pragmatic review of the literature [Date: 21 August 2020].

different interpretations from a philosophical perspective, including arguing the basis of Hill's criteria when classifying specific variables into independent (predictors) and dependent (outcomes). Furthermore, the multivariate statistical analyses have inherent limitations of their own as per the aphorism of the renowned British statistician George Box, "All models are wrong, but some are useful" [5]. There are also implicit constraints of the statistical packages, SPSS included, when it comes to loading a specific type or a count of variables into a model, including multiple regression, univariate and multivariate analysis of variance, supervised neural networks, and cluster analysis. For example, one may argue that the pterygomaxillary region is of utmost surgical importance to faciomaxillary surgeries, including reconstructive surgeries, and taking all the anthropometric points, lines, and angles into account is mandatory. However, that will render the data analyses too complicated to be executed, especially in connection with the multivariate and neural networks of the third hypoth- esis. Future research can consider the reciprocation of an abundance of anthropometrics and morphometrics while using advanced statistical packages running on a powerful supercomputer.

Nevertheless, we opine that our study is novel and should be impactful as a base for follow-up research. On 21 August 2020, we scraped the surface web by conducting a systematic review of the medical databases of literature, using Medical Subject Headings (MeSH) and generic keywords, to detect publications of relevance to the present study (Fig. 3). We searched the PubMed (the United States National Library of Medicine), Embase (Elsevier Database), and the Cochrane Library (the Cochrane Database of Systematic Reviews). We deployed a composite of MeSH-based keywords and generic terms, in addition to truncations, and Boolean operators as well. We included keywords related to six main themes, including morphometry, osteology, population demographics, applications in medicine, non-Bayesian statistics, and artificial intelligence. Our systematic review strat- 
egy generated a total count of $44,432,018$ papers distributed to PubMed (22,908,863, 51.56\%), Embase $(21,492,621,48.37 \%)$, and the Cochrane Library $(30,534,0.07 \%)$. The combination of all themes of keywords retrieved 961 publications, allocated to PubMed $(747,77.73 \%)$ and the Cochrane Library (214, $22.27 \%$ ) (Fig. 3). Hence, most of the publications were indexed in the PubMed database and Embase. However, Embase represented a lot of "noise" with publications that were not relevant to our study, its objectives, and methodology. Using the combination of themes, we filtered the search volume indexed in PubMed (747 publications) down by one-third of the original search volume (518 publications), by limiting the search results to articles written strictly in English, involving human species only, and the adults' age group (19+ years). We retrieved the full-text articles and scanned through the titles and abstracts, and we found very few articles that are relevant to the current study. Nonetheless, we concluded that our study is novel and the first of its kind, given its distinct objectives and pragmatic methodology, using an elaborate array of non-Bayesian statistics in parallel with machine learning.

Future studies should incorporate a larger sample size with a male-to-female ratio that is representative of the underlying population. Researchers can also attempt to use state-of-the-art equipment for the pursuit of exquisite morphometry, including the use of more advanced Vernier calliper and protractor that have fewer measurement errors. Besides, morphometry enthusiasts can record additional parameters, including surface areas and volumetrics, to enhance the accuracy and the predictive power of statistical as well as machine learning models [6]. Overcoming the limitations of data analytics, machine learning algorithms, and the statistical package can also serve as a leverage for reliable subsequent studies in line with the methodology of the present study $[3,7,9]$. Researchers should attempt to study osteology as well as radiology-based samples and compare the results from each to contrast premortem and postmortem specimens. They should also study and compare different ethnicities, age groups, patterns of lateralization of brain functions for the potential modifier effect on the stomatognathic system $[8,10]$. Research can involve individuals with pathologies and conditions of interest, for example, those with alveolar osteitis, pterygoid implants, dental prostheses, and ailments that can affect bone density and microstructure. We encourage conducting studies that are not purely observational, studies of quasi-experimental design, randomized case-control trials, aggregate studies, and meta-analytic studies of the supreme level-of-evidence [38].

\section{CONCLUSIONS}

Significant sexual, as well as laterality-based dimorphism, and bivariate correlations, existed. For the first hypothesis, significant sexual dimorphism existed favouring males and as per the pre-study anticipation. However, these were in connection with two morphometric parameters only, including $D$ [greater palatine foramen] and D [spinosum]. For the second hypothesis, significant laterality-based dimorphism was more diversified as it did not favour one side of the skull even when stratifying the sample based on sex, and it involved more morphometric parameters including D [ $x-y$ inclin.], D [x-z inclin.], D [hamulus], and $D$ [spinosum]. Each of sexual and laterality-based dimorphism had a medium effect size. Significant intra-variable and inter-variable correlations existed for the whole sample. However, contrary to the expectations, these correlations involved few morphometric parameters and had a weak-to-medium effect size. Testing the third hypothesis using the univariate analysis of variance failed to detect any significant predictors. On the contrary, multivariate statistical models were triumphant in elucidating the significant effect of laterality, and the interaction of sex and laterality on the inclination in the parasagittal and coronal planes for $D$ [prime], respectively. These results were consilient with the alternative hypothesis in connection with causality relationships. The weak effect size for those explanatory variables entails covert predictors and perhaps an abundance of them interacting to manifest the total variance within the outcome variables. Supervised and unsupervised machine learning, using neural networks and clustering analysis, reconciled with the non-Bayesian statistical models, especially the multivariate tests, and across all of the three hypotheses. The k-means cluster analysis highlighted the significant classifier effect of D [prime], its 3D inclination, and D [hamulus].

Future studies should incorporate a larger sample size with a male-to-female ratio representing the underlying population. Researchers can also attempt to use state-of-the-art equipment to pursue exquisite morphometry, including the use of Vernier calliper and protractor that have fewer measurement errors. Clinical researchers should explore the rele- 
vant morphometry of the maxillary sinus, including its mucosal lining, also known as the Schneiderian membrane, which is of prime importance for maxillofacial surgeons and implant dentists. Morphometry enthusiasts can record additional parameters, including surface areas and volumetrics, to enhance the accuracy, predictive power, and machine learning models. Researchers should attempt to study osteology and radiology-based samples, and compare the results from each to contrast premortem and postmortem specimens. Researchers should also study and compare different ethnicities, age groups, patterns of lateralization of brain functions for the potential modifier effect on the stomatognathic system.

\section{Availability of data}

Our data, including the raw dataset, are available upon request from the corresponding author.

\section{Conflict of interest: None declared}

\section{REFERENCES}

1. Albrektsson $T$, Zarb $G$, Worthington $P$, et al. The long-term efficacy of currently used dental implants: a review and proposed criteria of success. Int J Oral Maxillofac Implants. 1986; 1(1): 11-25, indexed in Pubmed: 3527955.

2. Al-Imam A, Al-Khazraji A. Alveolar osteitis following tooth extraction: systematic review and google trends analysis instigated by the first upper premolar case reported from Iraq. Modern Applied Science. 2019; 13(3): 1, doi: 10.5539/mas.v13n3p1.

3. Al-Imam A, Al-Lami F. Machine learning for potent dermatology research and practice. J Dermatol Dermatol Surg. 2020; 24(1): 1-4, doi: 10.4103/jdds.jdds_54_19.

4. Al-Imam A, Motyka M, Jędrzejko M. Conflicting opinions in connection with digital superintelligence. IAES Int J Artificial Intelligence (IJ-AI). 2020; 9(2): 336-348, doi: 10.11591/ijai.v9.i2.pp336-348.

5. Al-Imam A, Sahai A, Al-Derzi AR, et al. All Models Are Wrong, But Some Are Useful: On the Non-Bayesian Statistical Robustness of Hilton's Law. Eur J Anat. 2020; 24(1): 75-78.

6. Al-Imam A, Sahai A, Al-Nuaimi S, et al. The articular surfaces of the proximal segment of ulna: Morphometry and morphomechanics based on digital image analysis and concepts of fractal geometry. J Anat Soc India. 2018; 67(1): 40-49, doi: 10.1016/j.jasi.2018.04.003.

7. Al-Imam A. A novel method for computationally efficacious linear and polynomial regression analytics of big data in medicine. Modern Applied Science. 2020; 14(5): 1-10, doi: 10.5539/mas.v14n5p1.

8. Al-Imam A. Lateralization of brain functions: a randomized single-blinded observational study. Iran J Psych Behav Sci. 2018; 12(1), doi: 10.5812/ijpbs.8074.

9. Al-Imam A. Optimizing linear models via sinusoidal transformation for boosted machine learning in medicine. J Faculty Med Baghdad. 2019; 61(3/4): 128-136.
10. Al-Imam A. The relation between cerebral dominance and visual analytic skills in Iraqi Medical students, a cross sectional analysis. J Anat Soc India. 2017; 66: S42-S43, doi: 10.1016/j.jasi.2017.08.136.

11. Al-Imam, A. Inferential analysis of big data in real-time: one giant leap for spatiotemporal digital epidemiology in dentistry. Odontostomatol Res Anat Learning a Implantology. 2019; 12(1): 1-14.

12. Balaji VR, Lambodharan R, Manikandan D, et al. Pterygoid implant for atrophic posterior maxilla. J Pharm Bioallied Sci. 2017; 9(Suppl 1): S261-S263, doi: 10.4103/jpbs. JPBS_103_17, indexed in Pubmed: 29284976.

13. Bastir M, Rosas A. Comparative ontogeny in humans and chimpanzees: Similarities, differences and paradoxes in postnatal growth and development of the skull. Ann Anat - Anat Anz. 2004; 186(5-6): 503-509, doi: 10.1016/ s0940-9602(04)80096-7.

14. Bastir M, Rosas A. Facial heights: evolutionary relevance of postnatal ontogeny for facial orientation and skull morphology in humans and chimpanzees. J Hum Evol. 2004; 47(5): 359-381, doi: 10.1016/j.jhevol.2004.08.009, indexed in Pubmed: 15530353.

15. Bidra AS, Huynh-Ba G. Implants in the pterygoid region: a systematic review of the literature. Int J Oral Maxillofac Surg. 2011; 40(8): 773-781, doi: 10.1016/j. ijom.2011.04.007, indexed in Pubmed: 21601424.

16. Bienek DR, Filliben JJ. Risk assessment and sensitivity meta-analysis of alveolar osteitis occurrence in oral contraceptive users. J Am Dent Assoc. 2016; 147(6): 394-404, doi: 10.1016/j.adaj.2016.01.011, indexed in Pubmed: 27017181.

17. Blum IR. Contemporary views on dry socket (alveolar osteitis): a clinical appraisal of standardization, aetiopathogenesis and management: a critical review. Int J Oral Maxillofac Surg. 2002; 31(3): 309-317, doi: 10.1054/ ijom.2002.0263, indexed in Pubmed: 12190139.

18. Candel E, Peñarrocha D, Peñarrocha M. Rehabilitation of the atrophic posterior maxilla with pterygoid implants: a review. J Oral Implantol. 2012; 38 Spec No: 461-466, doi: 10.1563/AAID-JOI-D-10-00200, indexed in Pubmed: 21568718.

19. Cardini A, Polly PD, Cardini A, et al. Larger mammals have longer faces because of size-related constraints on skull form. Nat Commun. 2013; 4(1): 2458, doi: 10.1038/ ncomms3458, indexed in Pubmed: 24045342.

20. Čechová M, Dupej J, Brůžek J, et al. Sex estimation using external morphology of the frontal bone and frontal sinuses in a contemporary Czech population. Int J Legal Med. 2019; 133(4): 1285-1294, doi: 10.1007/s00414-01902063-8, indexed in Pubmed: 30982130.

21. Cox LA. Modernizing the Bradford Hill criteria for assessing causal relationships in observational data. Crit Rev Toxicol. 2018; 48(8): 682-712, doi: 10.1080/10408444.2018.1518404, indexed in Pubmed: 30433840.

22. Crawford JY. Dry socket. Dent Cosmos. 1896; 38: 929.

23. Daly B, Sharif MO, Newton T, et al. Local interventions for the management of alveolar osteitis (dry socket). Cochrane Database Syst Rev. 2012; 12: CD006968, doi: 10.1002/14651858. CD006968.pub2, indexed in Pubmed: 23235637.

24. Durieux N, Vandenput S, Pasleau F. [OCEBM levels of evidence system]. Rev Med Liege. 2013; 68(12): 644-649, indexed in Pubmed: 24564030. 
25. Erdogan N, Unur E, Baykara M. CT anatomy of pterygopalatine fossa and its communications: a pictorial review. Comput Med Imaging Graph. 2003; 27(6): 481-487, doi: 10.1016/ s0895-6111(03)00038-7, indexed in Pubmed: 14575781.

26. Hadžiselimović $H$, Čuš $M$. The appearance of internal structures of the brain in relation to configuration of the human skull. Cell Tiss Org. 1966; 63(3): 289-299, doi: 10.1159/000142794.

27. Kiliaridis S. Masticatory muscle influence on craniofacial growth. Acta Odontol Scand. 2009; 53(3): 196-202, doi: 10.3109/00016359509005972.

28. Kim SW, Nishimura J, Tsuchiya A. Expanding (3+1)-dimensional universe from a lorentzian matrix model for superstring theory in $(9+1)$ dimensions. Phys Rev Lett. 2012; 108(1): 011601, doi: 10.1103/PhysRevLett.108.011601, indexed in Pubmed: 22304254.

29. Kozerska M, Skrzat J, Szczepanek A. Application of the temporal bone for sex determination from the skeletal remains. Folia Med Cracov. 2015; 55(2): 33-39, indexed in Pubmed: 26839241.

30. Lieberman DE. Sphenoid shortening and the evolution of modern human cranial shape. Nature. 1998; 393(6681): 158-162, doi: 10.1038/30227, indexed in Pubmed: 9603517.

31. Lofgren RH. Surgery of the pterygomaxillary fossa. Arch Otolaryngol. 1971; 94(6): 516-524, doi: 10.1001/ archotol.1971.00770070816006, indexed in Pubmed: 5129223.

32. Manoonpol C, Plakornkul V. Sex determination using mastoid process measurement in Thais. J Med Assoc Thai. 2012; 95(3): 423-429, indexed in Pubmed: 22550843.

33. Marcussen KB, Laulund AS, Jørgensen HL, et al. A Systematic Review on Effect of Single-Dose Preoperative Antibiotics at Surgical Osteotomy Extraction of Lower Third Molars. J Oral Maxillofac Surg. 2016; 74(4): 693-703, doi: 10.1016/j.joms.2015.11.017, indexed in Pubmed: 26706491.

34. McBratney-Owen B, Iseki S, Bamforth SD, et al. Development and tissue origins of the mammalian cranial base. Dev Biol. 2008; 322(1): 121-132, doi: 10.1016/j. ydbio.2008.07.016, indexed in Pubmed: 18680740.

35. Møller M. A scaled conjugate gradient algorithm for fast supervised learning. Neural Networks. 1993; 6(4): 525-533, doi: 10.1016/s0893-6080(05)80056-5.

36. Montgomery WW, Katz R, Gamble JF. Anatomy and surgery of the pterygomaxillary fossa. Ann Otol Rhinol Laryngol. 1970; 79(3): 606-618, doi: 10.1177/000348947007900326, indexed in Pubmed: 5426889

37. Musilová B, Dupej J, Velemínská J, et al. Exocranial surfaces for sex assessment of the human cranium. Forensic Sci Int. 2016; 269: 70-77, doi: 10.1016/j.forsciint.2016.11.006, indexed in Pubmed: 27883982.

38. Oxford Centre for Evidence-Based Medicine. OCEBM Levels of Evidence. https://www.cebm.net/2016/05/ocebm-levels-of-evidence/ (accessed 3 September 2020).

39. Pauler D. The Schwarz criterion and related methods for normal linear models. Biometrika. 1998; 85(1): 13-27, doi: 10.1093/biomet/85.1.13.

40. Pogrel MA, Kaplan MJ. Surgical approach to the pterygomaxillary region. J Oral Maxillofac Surg. 1986; 44(3):
183-187, doi: 10.1016/0278-2391(86)90105-9, indexed in Pubmed: 3456438.

41. Polanski JM, Franciscus RG. Patterns of craniofacial integration in extant Homo, Pan, and Gorilla. Am J Phys Anthropol. 2006; 131(1): 38-49, doi: 10.1002/ajpa.20421, indexed in Pubmed: 16552733.

42. Ramamoorthy B, Pai MM, Prabhu LV, et al. Assessment of craniometric traits in South Indian dry skulls for sex determination. J Forensic Leg Med. 2016; 37: 8-14, doi: 10.1016/j. jflm.2015.10.001, indexed in Pubmed: 26519924.

43. Rodríguez X, Rambla F, De Marcos Lopez L, et al. Anatomical study of the pterygomaxillary area for implant placement: cone beam computed tomographic scanning in 100 patients. Int J Oral Maxillofac Implants. 2014; 29(5): 1049-1052, doi: 10.11607/jomi.3173, indexed in Pubmed: 25216128.

44. Sánchez J, Calvo V, Sánchez A, et al. Sensitization to 10 mites in a tropic area. Der $p$ and Der $f$ are important risk factor for sensitization to other mites from Pyroglyphidae, Acaridae, Chortoglyphidae, and Glyciphagidae families. Rev Alerg Mex. 2017; 64(2): 153-162, doi: 10.29262/ram. v64i2.243, indexed in Pubmed: 28658723.

45. Schünemann $H$, Hill $S$, Guyatt $G$, et al. The GRADE approach and Bradford Hill's criteria for causation. J Epidemiol Community Health. 2011; 65(5): 392-395, doi: 10.1136/ jech.2010.119933, indexed in Pubmed: 20947872.

46. Simental AA, Johnson JT, Myers EN. Cervical metastasis from squamous cell carcinoma of the maxillary alveolus and hard palate. Laryngoscope. 2006; 116(9): 1682-1684, doi: 10.1097/01.mlg.0000233607.41540.28, indexed in Pubmed: 16955004.

47. Taberner-Vallverdú M, Sánchez-Garcés MÁ, Gay-Escoda C. Efficacy of different methods used for dry socket prevention and risk factor analysis: A systematic review. Med Oral Patol Oral Cir Bucal. 2017; 22(6): e750-e758, doi: 10.4317/medoral.21705, indexed in Pubmed: 29053647.

48. Toneva D, Nikolova S, Harizanov S, et al. Sex estimation by size and shape of foramen magnum based on CT imaging. Leg Med (Tokyo). 2018; 35: 50-60, doi: 10.1016/j. legalmed.2018.09.009, indexed in Pubmed: 30268691.

49. Urban JE, Weaver AA, Lillie EM, et al. Evaluation of morphological changes in the adult skull with age and sex. J Anat. 2016; 229(6): 838-846, doi: 10.1111/joa.12247, indexed in Pubmed: 25406956.

50. Yengopal V, Mickenautsch S. Chlorhexidine for the prevention of alveolar osteitis. Int J Oral Maxillofac Surg. 2012; 41(10): 1253-1264, doi: 10.1016/j.ijom.2012.04.017, indexed in Pubmed: 22622144.

51. Zelditch M, Fink W. Heterochrony and heterotopy: stability and innovation in the evolution of form. Paleobiology. 2015; 22(2): 241-254, doi: 10.1017/ s0094837300016195.

52. Zelditch $M$, Sheets $H$, Fink W. The ontogenetic dynamics of shape disparity. Paleobiology. 2016; 29(1): 139-156, doi: 10.1666/0094-8373(2003)029<0139:todosd >2.0.co;2.

53. Zhou S, Zhang T, Peng Bo, et al. Targeted delivery of epirubicin to tumor-associated macrophages by sialic acid-cholesterol conjugate modified liposomes with improved antitumor activity. Int J Pharm. 2017; 523(1): 203-216, doi: 10.1016/j.ijpharm.2017.03.034, indexed in Pubmed: 28336455. 